Revue de recherche interdisciplinaire sur le genre et la sexualité

35 | 2018

Striges en tous genres

\title{
La compassion pour soi-même peut-elle protéger des conséquences néfastes liées à une image corporelle négative?
}

Robin Wollast

\section{OpenEdition}

12 Journals

Édition électronique

URL : https://journals.openedition.org/sextant/401

DOI : 10.4000/sextant.401

ISSN : 2795-8736

Éditeur

Éditions de l'Université de Bruxelles

Édition imprimée

Date de publication : 1 décembre 2018

Pagination : 117-129

ISBN : 978-2-8004-1636-6

ISSN : 1370-267X

\section{Référence électronique}

Robin Wollast, « La compassion pour soi-même peut-elle protéger des conséquences néfastes liées à une image corporelle négative? », Sextant [En ligne], 35 | 2018, mis en ligne le 01 novembre 2021, consulté le 08 décembre 2021. URL : http://journals.openedition.org/sextant/401 ; DOI : https:// doi.org/10.4000/sextant.401

\section{ल(๑)}

La revue Sextant est mise à disposition selon les termes de la Licence Creative Commons Attribution Pas d'Utilisation Commerciale - Partage dans les Mêmes Conditions 4.0 International. 


\title{
La compassion pour soi-même peut-elle protéger des conséquences néfastes liées à une image corporelle négative?
}

\author{
Robin WoLLAST
}

\begin{abstract}
Aucune grâce extérieure n'est complète si la beauté intérieure ne la vivifie. La beauté de l'âme se répand comme une lumière mystérieuse sur la beauté du corps. Victor Hugo, Post-scriptum de ma vie.
\end{abstract}

L'apparence physique est le premier élément que l'on remarque chez une personne. Elle permet de se forger rapidement une opinion, un jugement ou une impression, à partir du style vestimentaire, de la physionomie, de la posture générale, de la couleur de peau ou encore du maintien d'un individu. Il est dès lors possible d'attribuer à cette personne une appartenance sociale ou un environnement familial sur la base des informations observées. Bien que les apparences puissent être trompeuses, les médias usent et abusent au quotidien de l'image du corps ${ }^{1}$. En effet, ceux-ci (télévision, magazines, internet, jeux vidéo, etc.) imposent les normes de beauté en véhiculant des images sexualisées, idéalisées et stéréotypées des femmes et des hommes, ce qui n'est pas sans conséquence pour le grand public ${ }^{2}$. L'impossibilité pour une personne de ressembler ou de s'identifier à une construction inatteignable ou à un modèle parfait (comme les canons de beauté) peut avoir des conséquences négatives pour elle : c'est ce qu'on appelle la tyrannie de l'apparence ${ }^{3}$. Le présent article répond à deux objectifs complémentaires. Primo, il sensibilise le lecteur à la théorie de l'objectification qui décrit l'influence de cette représentation omniprésente et idéalisée de la beauté féminine sur la santé mentale des femmes. Secundo, la pertinence de cet article se justifie par son apport théorique important concernant la compréhension du rôle que l'auto-compassion peut présenter dans la perception que se font les femmes de leur

${ }^{1}$ A. Dworkin, "Against the male flood : Censorship, pornography, and equality », in D. CoRnell (éd.), Oxford readings in feminism : Feminism and pornography, Oxford, Oxford University Press, 2000.

${ }^{2}$ N. Wolf, The beauty myth : How images of beauty are used against women, New York, William Morrow and Company, 1991.

3 J.-Fr. Amadieu, Le poids des apparences. Beauté, amour et gloire, Paris, Odile Jacob, 2002. 
propre corps. A ce jour, les travaux portant sur les liens entre l'image du corps et la compassion pour soi sont rares et mériteraient pourtant d'être exploités. De fait, cet article théorique propose une piste de réflexion nouvelle, nécessaire et encourageante, sur le rôle protecteur que peut avoir la compassion pour soi face aux conséquences néfastes liées à une image corporelle négative.

\section{L'objectification sexuelle}

Le phénomène d'objectification apparaît dès que les individus sont traités comme s'ils ne présentaient aucun état mental ni aucune valeur morale ou quand certaines caractéristiques humaines sont niées (l'autonomie, la subjectivité, l'agentivité ${ }^{4}$, par exemple ${ }^{5}$ ). Selon le philosophe Emmanuel $\mathrm{Kant}^{6}$, le risque d'objectification est présent dans toutes les rencontres sexuelles lorsqu'un des protagonistes ne peut devenir qu'un simple objet de satisfaction ou d'appétit ${ }^{7}$ pour l'autre, dès que l'instrumentalisation du corps prend place, dissociant le corps et l'esprit et engendrant une perte d'humanité ${ }^{8}$. En somme, le processus d'objectification s'installe lorsque les parties ou les fonctions du corps d'une personne sont séparées et réduites à un statut d'instrument ou si elles sont vues comme capables de représenter la personne dans sa totalité ${ }^{9}$. En ce sens, la théorie de l'objectification développée par Fredrickson et Roberts permet d'expliquer l'impact de l'objectification sur la santé mentale des femmes et, par conséquent, l'une des raisons pour lesquelles les femmes ont souvent tendance à développer un sentiment d'insatisfaction par rapport à leur propre corps ${ }^{10}$. Cette théorie postule que la société actuelle génère un contexte dans lequel prend place l'objectification du corps de la femme : celui-ci est alors perçu comme un objet qui peut être regardé et évalué par autrui. Selon cette théorie, ce phénomène d'objectification sexuelle (tendance à considérer ou à traiter une personne comme un objet sexuel, comme un corps à consommer ${ }^{11}$ ) ou de sexualisation peut amener les femmes à intérioriser le regard qu'autrui porte sur elles : il s'agit du phénomène d'auto-objectification. L'omniprésence de cette représentation idéalisée de la femme, les amène à adopter le point de vue d'un observateur sur leur propre corps, à « s'autoobjectiver », impliquant ainsi des questions du type « De quoi ai-je l'air ? », avec un

${ }^{4}$ M. Nussbaum, « Objectification », Philosophy and Public Affairs, 24, 1995, p. 249-291.

${ }^{5}$ Voir pour une taxonomie plus détaillée, J. M. LACroix et F. PratTo, «Instrumentality and the denial of personhood: The social psychology of objectifying others ", Revue internationale de psychologie sociale, 28/1, 2015, p. 183-211.

6 E. Kant, Fondements de la métaphysique des mours, traduction de Victor Delbos, revue par A. Philonenko, ... avec une introduction et des notes nouvelles, Paris, J. Vrin, 1992.

7 E. L. PAPADaki, "Sexual objectification : From Kant to contemporary feminism », Contemporary Political Theory, 6/3, 2007, p. 330-348.

8 E. KANT, Leçons d'éthique, présentation, traduction et notes par Luc LANGLOIs, Paris, Librairie générale française, 1997.

${ }^{9}$ S. L. BARTKY, Femininity and domination; studies in the phenomenology of oppression, New York, Routledge, 1990, p. 26.

${ }^{10}$ B. L. FredricKSON et T. A. RoberTs, « Objectification theory : Toward understanding women's lived experiences and mental health risks », Psychology of Women Quarterly, 21/2, 1997, p. 173-206.

${ }^{11}$ S. L. BARTKY, Femininity and domination, op. cit. 
focus orienté sur l'apparence plutôt que sur la compétence ${ }^{12}$. En d'autres termes, les femmes peuvent être amenées à considérer elles-mêmes leur corps comme un objet qui peut être regardé, évalué, jugé, désiré ou détesté par les autres.

Les recherches scientifiques mettent en évidence que ce phénomène d'autoobjectification concerne aussi les hommes ${ }^{13}$, même si des attributs corporels différents sont impliqués en fonction du genre ${ }^{14}$. En effet, la littérature démontre que les médias génèrent un environnement sexuellement objectifiant qui favorise surtout l'idéal de musculation pour les hommes ${ }^{15}$ et l'idéal de minceur chez les femmes ${ }^{16}$. En conséquence, ces images renforcent les rôles traditionnels des genres comme celui de l'homme dominant, viril et actif face à une femme soumise, féminine, passive et fragile, alimentant dès lors l'expression des inégalités de genre ${ }^{17}$. Par conséquent, l'exposition aux images objectifiantes alimente les attitudes sexistes chez les individus comme le sexisme hostile (" attitude antagoniste vis-à-vis des femmes qui sont perçues comme usurpant le pouvoir des hommes ») ou bienveillant («attitude sexiste plus implicite, teintée de chevalerie, qui a une apparence anodine et qui semble même différencier favorablement les femmes en les décrivant comme chaleureuses et sociables ") ${ }^{18}$. En outre, l'objectification sexuelle renforce l'adhésion au mythe du viol ${ }^{19}$. En d'autres termes, dans un contexte social et médiatique où les violences

${ }^{12}$ Pour une contribution théorique majeure voir : D. M. Szymanski, L. B. Moffitt, et E. R. CARR, "Sexual Objectification of Women : Advances to Theory and Research », The Counseling Psychologist, 39/1, 2010, p. 6-38.

${ }^{13}$ P. Strelan et D. Hargreaves, « Women Who Objectify Other Women : The Vicious Circle of Objectification? », Sex Roles, 52/9-10, 2005, p. 707-712.

${ }^{14}$ R. A. Leit, H. G. Pope et J. J. GraY, « Cultural expectations of muscularity in men : The evolution of Playgirl centerfolds », International Journal of Eating Disorders, 29/1, 2001, p. 90-93.

${ }^{15}$ S. Daniel, S. K. Bridges et M. P. Martens, « The Development and Validation of the Male Assessment of Self-Objectification (MASO) », Psychology of Men \& Masculinity, 15/1, $78,2014$.

${ }^{16}$ P. J. Johnson, D. R. McCreary et J. S. Mills, « Effects of exposure to objectified male and female media images on men's psychological well-being ", Psychology of Men \& Masculinity, 8, 2007, p. 95-102.

17 V. Swami et M. VoraceK, « Associations among men's sexist attitudes, objectification of women, and their own drive for muscularity », Psychology of Men \& Masculinity, 14, 2012, p. $168-174$.

${ }^{18}$ P. Glick et S. T. FISKE, « The ambivalent sexism inventory : Differentiating hostile and benevolent sexism ", Journal of Personality and Social Psychology, 70/3, 491, 1996 ; B. Dardenne, N. Delacollette, C. GréGoire, et D. LecocQ, « Structure latente et validation de la version française de l'Ambivalent Sexism Inventory : l'échelle de sexisme ambivalent", L'Année psychologique, 106/2, 2006, p. 235-263 ; V. Swami, R. Coles, E. Wilson, N. Salem, K. Wyrozumska et A. Furnham, « Oppressive beliefs at play : Associations among beauty ideals and practices and individual differences in sexism, objectification of others, and media exposure ", Psychology of Women Quarterly, 34/3, 2010 ; M. CikARA, J. L. Eberhardt et S. T. FISKE, "From agents to objects : Sexist attitudes and neural responses to sexualized targets ", Journal of Cognitive Neuroscience, 23, 2010, p. 540-551.

19 S. Loughnan, A. Pina, E. Vasquez et E. Puvia, « Sexual objectification increases rape victim blame and decreases perceived suffering », Psychology of Women Quarterly, 37/4, 2013, 
sexuelles sont justifiées, banalisées ou socialement acceptées, les individus ont davantage tendance à responsabiliser, blâmer et culpabiliser les femmes victimes de viol sur la base de leur apparence ${ }^{20}$ (" Pas étonnant qu'elle se soit fait violer, tu as vu comment elle est habillée ? »). C'est ce qu'on appelle, la culture du viol ${ }^{21}$ : un phénomène répandu dans notre société. En plus des associations morbides qu'elle entretient avec le sexisme, l'objectification sexuelle influe considérablement sur la santé mentale des individus et en particulier sur celle des femmes.

\section{Les conséquences négatives de l'objectification sexuelle}

Fredrickson et Roberts mettent en évidence plusieurs conséquences néfastes de cette auto-objectification ${ }^{22}$. L'impossibilité consciente ou inconsciente pour une personne de ressembler ou de s'identifier aux normes de beauté imposées par la société peut avoir des conséquences néfastes comme la honte ressentie par rapport à son propre corps ou une augmentation de l'angoisse liée à son apparence physique, l'oppression qui nous étreint quand notre corps est regardé et évalué. Par la suite, de nombreuses études ${ }^{23}$ ont démontré que l'auto-objectification génère des conséquences psychologiques dommageables telles qu'une baisse de l'estime de soi, une réduction des états émotionnels, une baisse des performances cognitives, une perception plus faible des sensations internes, un affaiblissement de la santé physique et des problèmes au niveau de la santé mentale, comme des troubles alimentaires, de la dépression ou encore des dysfonctionnements sexuels ${ }^{24}$.

Corollairement, McKinley et Hyde décrivent les mécanismes sous-jacents liés à l'auto-objectification et les effets que peut engendrer l'internalisation des standards de

p. $455-461$.

${ }^{20}$ P. Bernard, D. Loughnan, C. Marchal, A. Godart et O. Klein, « The Exonerating Effect of Sexual Objectification: Sexual Objectification Decreases Rapist Blame in a Stranger Rape Context », Sex roles, 72/11-12, 2015, p. 499-508.

${ }^{21}$ N. Connell et C. Wilson (éd.), Rape : the first sourcebook for women, New York et Scarborough, Plume, 1974.

${ }^{22}$ B. L. Fredrickson et T. A. RoberTs, « Objectification theory: Toward understanding women's lived experiences and mental health risks », op. cit.

${ }^{23}$ Voir par exemple : B. L. Fredrickson, T.-A. Roberts, S. M. Noll, D. M. QuinN et J. M. Twenge, « That swimsuit becomes you : Sex differences in self-objectification, restrained eating, and math performance », Journal of Personality and Social Psychology, 75/5, 1998 ; D. M. Quinn, R. W. Kallen et C. CatheY, « Body on my mind : The lingering effect of state self-objectification », Sex Roles, 55/11-12, 2006, p. 869-874 ; D. L. FIISSEL et K. D. LAFRENIERE, «Weight control motives for cigarette smoking : further consequences of the sexual objectification of women ? », Feminism \& Psychology, 16/3, 2006, p. 327-344 ; J. J. Muehlenkamp et R. N. Saris-Baglama, « Self-objectification and its psychological outcomes for college women ", Psychology of Women Quarterly, 26/4, 2002, p. 371-379 ; D. T. SANChEZ et A. K. KIEFER, « Body concerns in and out of the bedroom : Implications for sexual pleasure and problems », Archives of Sexual Behavior, 36/6, 2007, p. 808-820.

24 Pour une revue de la littérature, voir B. Moradi et Y. P. Huang, « Objectification theory and psychology of women: A decade of advances and future directions ", Psychology of Women Quarterly, 32/4, 2008, p. 377-398. 


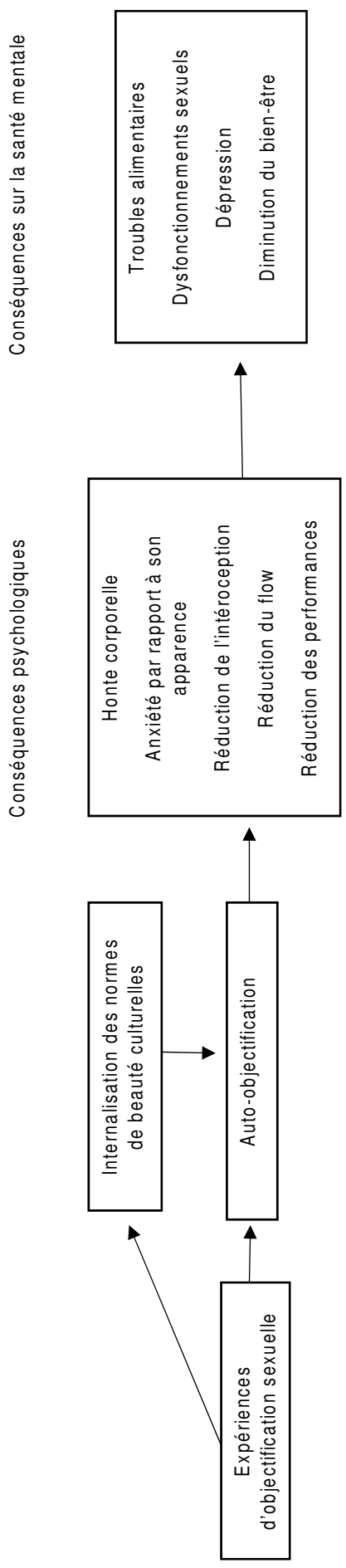

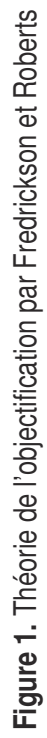


beauté chez les femmes ${ }^{25}$. En accord avec ces hypothèses, certaines femmes peuvent croire qu'atteindre un modèle de beauté inaccessible est possible, ce qui les pousse à se focaliser davantage sur leur apparence. Ces chercheures proposent trois dimensions : l'auto-surveillance, la honte corporelle et le sentiment de contrôle. Dans la première, les femmes s'engagent dans des processus d'auto-surveillance de leur propre corps, perçu comme un objet, en tant qu'observatrices extérieures capables de juger leur apparence en se comparant à la norme de beauté, mais aussi dans leurs interactions interpersonnelles (avec les pairs par exemple). Dans la deuxième, les femmes peuvent être amenées à ressentir un sentiment de honte ou d'inconfort quand elles réalisent qu'elles n'ont pas réussi à atteindre leur idéal. Enfin, dans la troisième, certaines femmes entretiennent la croyance qu'elles peuvent contrôler leur image corporelle. Ce sentiment de contrôle peut les amener à se sentir responsables de la manière dont elles perçoivent leur corps. En effet, suite à cette auto-objectification, la réaction des femmes consiste souvent à se contrôler davantage en accordant une attention extrême à leur apparence, à leurs vêtements, à leur coiffure ou à leur maquillage. Mais aussi, en s'imposant un contrôle alimentaire strict ou de l'exercice physique pratiqué de manière intensive pour être satisfaites de leur image et orienter la perception $\mathrm{du}$ regard des autres sur elles-mêmes. Elles espèrent ainsi améliorer leur qualité de vie. De fait, ce sentiment de contrôle peut contribuer à diminuer leur sentiment de stress à l'égard des normes de beauté, mais aussi engendrer un sentiment d'autocritique plus fort lorsqu'elles ne sont pas satisfaites de leurs efforts. En ce sens, la littérature actuelle considère l'auto-surveillance ou surveillance du corps comme la première manifestation liée à l'auto-objectification ${ }^{26}$.

La théorie de l'objectification propose deux types d'auto-objectifiation. La première est l'auto-objectification « trait », ou dispositionnelle, qui témoigne des différences individuelles dans la manière de percevoir son apparence. Certains individus accordent plus d'importance à leur image que d'autres. La seconde est l'auto-objectification "état » qui correspond au fait que l'auto-objectification peut être amorcée ou amplifiée dans certains contextes (dans la rue, à la plage, dans les discothèques, dans l'intimité sexuelle, ...). Une étude a ainsi montré que les femmes recevaient davantage de remarques évaluatives à caractère sexuel et objectificant dans les lieux publics et mixtes ${ }^{27}$. Dès lors, l'auto-objectification serait plus forte dans les situations où les individus auraient conscience du regard des autres sur leur corps.

${ }^{25}$ N. M. MCKINLEY et J. S. HYDE, « The objectified body consciousness scale development and validation », Psychology of Women Quarterly, 20/2, 1996, p. 181-215.

${ }^{26}$ N. M. MCKINLEY, "Women and objectified body consciousness : mothers' and daughters' body experience in cultural, developmental, and familial context », Developmental Psychology, 35/3, 1999 ; T. L. TyLKa et M. S. HiLL, "Objectification theory as it relates to disordered eating among college women », Sex Roles, 51/11-12, 2004, p. 719-730.

27 J. K. Swim, L. L. Hyers, L. L. Cohen et M. J. Ferguson, « Everyday Sexism : Evidence for Its Incidence, Nature, and Psychological Impact From Three Daily Diary Studies », Journal of Social Issues, 57/1, 2001, p. 31-53. 
Un an après le développement de la théorie de l'objectification, Fredrickson et ses collègues ${ }^{28}$ ont eu l'idée d'analyser ce concept d'auto-objectification («état »et «trait») de manière originale. Pour ce faire, elles ont demandé à leurs participants, hommes et femmes, d'enfiler soit un maillot de bain une pièce (condition d'objectification), soit un pull à col en $\mathrm{V}$ (condition contrôle), dans une cabine d'essayage individuelle où se trouvait un miroir. Une fois les participants habillés, ils devaient répondre à un questionnaire mesurant la honte par rapport au corps et résoudre des tests de mathématiques. Les résultats de l'étude ont démontré que les individus qui portaient un maillot ressentaient davantage de honte par rapport à leur corps que ceux qui portaient un pull à col en V. De plus, les femmes obtenaient des résultats aux tests mathématiques plus faibles lorsqu'elles étaient dans la condition d'induction d'un état d'auto-objectification (maillot) que dans la condition contrôle (pull). Chez les hommes, l'essai du maillot provoquait aussi une prise de conscience de la timidité ainsi qu'une diminution de la confiance en soi. En résumé, cette étude démontre que les individus portant un maillot de bain une pièce se définissent eux-mêmes à travers leur corps. Ce phénomène constitue ce que les chercheurs qualifient d'auto-objectification. En effet, les individus qui se retrouvent dans une position « objectifiante » ont plutôt tendance à se percevoir selon un point de vue à la troisième personne (" de quoi ai-je l'air ? ») plutôt qu'à la première personne (« de quoi suis-je capable ? », « comment est-ce que je me sens ? »). Cette étude renforce les hypothèses de la théorie de l'objectification selon lesquelles l'auto-objectification est liée à un sentiment de honte corporelle et est consommatrice de ressources attentionnelles. Par la suite, de nombreux chercheurs ont répliqué ces résultats à travers différentes manipulations impliquant, par exemple, la présence d'une caméra visible. Ils filmaient les participants pour qu'ils se sentent regardés afin de rendre le contexte d'auto-objectification plus saillant ${ }^{29}$. De même, ils pouvaient exposer des participants à des images sexuellement objectifiantes ${ }^{30}$ ou créer des environnements qui conditionnent la perception du regard (une pièce comportant un grand miroir versus une pièce vide ${ }^{31}$, par exemple), influençant dès lors la manière dont les participants allaient se comporter. A terme, les études sont concluantes : le contexte d'objectification sexuelle ou d'auto-objectification altère la santé mentale et surtout la manière dont les individus vont percevoir leur propre corps. Une question peut dès lors être posée : sommes-nous tous et toutes concernés par ce phénomène?

${ }^{28}$ B. L. Fredrickson, T.-A. Roberts, S. M. Noll, D. M. Quinn et J. M. Twenge, « That swimsuit becomes you », op. cit.

29 R. K. Gay, et E. CAStano, « My body or my mind : The impact of state and trait objectification on women's cognitive resources », European Journal of Social Psychology, 40/5, 2010, p. 695-703.

30 J. S. Aubrey, J. R. Henson, K. M. Hopper et S. E. Smith, « A picture is worth twenty words (about the self) : Testing the priming influence of visual sexual objectification on women's self-objectification », Communication Research Reports, 26/4, 2009, p. 271-284.

31 M. Tiggemann et M. Boundy, « Effect of environment and appearance compliment on college women's self-objectification, mood, body shame, and cognitive performance " , Psychology of Women Quarterly, 32/4, 2008, p. 399-405. 


\section{L'objectification, processus universel ou spécifique à un contexte culturel particulier ?}

Pour rappel, la théorie de l'objectification postule que la société occidentale actuelle objectifie sexuellement le corps de la femme. En effet, la grande majorité des recherches ont été conduites aux Etats-Unis et en Europe de l'Ouest. Plus tard, d'autres équipes de chercheurs ont étudié ce phénomène dans des contextes différents et mis en évidence que l'auto-objectification engendrait aussi des conséquences négatives chez des Afro-Américains, des Hispaniques et des Américains d'origine asiatique $^{32}$ et chez des personnes homosexuelles ${ }^{33}$. De plus, certaines études tendent à démontrer que les conséquences négatives de l'auto-objectification apparaissent également dans différents pays comme le Népal ${ }^{34}, 1^{1}$ Inde $^{35}$, la Thaïlande ${ }^{36}$, la Corée du Sud ${ }^{37}$ ou encore la Chine ${ }^{38}$. En somme, ces études suggèrent que le phénomène d'objectification sexuelle serait un processus universel plutôt que spécifique et limité à la seule société occidentale.

Comme nous venons de le voir, il est fort difficile de lutter contre l'objectification, omniprésente dans nos sociétés. En revanche, certains processus sont susceptibles de moduler ses conséquences négatives sur la santé mentale. La littérature sur le sujet montre en effet que l'auto-objectification aurait un effet général et des conséquences difficiles à éviter sur la santé mentale. On sait en définitive peu de choses sur les modérateurs de cet effet. Rares en effet sont les études qui ont examiné les facteurs qui permettraient de se prémunir de ces conséquences négatives. Or, comprendre les facteurs modérateurs des effets de l'auto-objectification sur la santé mentale nous permettrait de préciser davantage la théorie de l'objectification, voire d'identifier certaines catégories d'individus particulièrement à risque ou, au contraire, qui seraient protégés des conséquences de l'objectification. En ce sens, le deuxième objectif de notre article théorique consiste à expliciter le rapport entre l'auto-objectification et ses

${ }^{32}$ M. R. Hebl, « The Swimsuit Becomes Us All : Ethnicity, Gender, and Vulnerability to Self-Objectification », Personality and Social Psychology Bulletin, 30/10, 2004, p. 1322-1331.

${ }^{33}$ M. KozaK, H. Frankenhauser et T.-A. Roberts, « Objects of desire : Objectification as a function of male sexual orientation », Psychology of Men \& Masculinity, 10/3, 2009, p. 225-230.

34 M. Crawford, I.-C. Lee, G. Portnoy, A. Gurung, D. Khati, P. Jha et A. C. Regmi, « Objectified Body Consciousness in a Developing Country : A Comparison of Mothers and Daughters in the us and Nepal », Sex Roles, 60/3-4, 2008, p. 174-185.

35 S. Loughnan, S. Fernandez-Campos, J. Vaes, G. Anjum, M. Aziz, C. Harada, E. Holland, I. Singh, E. Puvia et K. Tsuchiya, « Exploring the role of culture in sexual objectification : A seven nations study », Revue internationale de psychologie sociale, 28/1, 2015, p. 125-152.

${ }^{36}$ R. Wollast, E. Puvia, P. Bernard, P. Tevichapong et O. Klein, « How Sexual Objectification Generates Dehumanization in Western and Eastern Cultures - a Comparison Between Belgium and Thailand », Swiss Journal of Psychology, 2018, 77/2, p. 69-82.

37 S. O. LyU, « Travel selfies on social media as objectified self-presentation », Tourism Management, 54, 2016, p. 185-195.

${ }^{38}$ F. Teng, K. T. Poon, H. Zhang, Z. Chen, Y. Yang, et X. Wang, « Situational cuing of materialism triggers self-objectification among women (but not men) : The moderating role of self-concept clarity », Personality and Individual Differences, 97, 2016, p. 220-228. 
conséquences sur la santé mentale, envisagé sous l'angle d'une stratégie de résilience : l'auto-compassion (capacité à être bienveillant et compréhensif avec soi-même ${ }^{39}$ ) constitue de ce point de vue un facteur lénifiant et protecteur qui aide l'individu à mieux affronter la menace que représente l'objectification.

\section{L'auto-compassion}

L'auto-compassion, ou la compassion pour soi-même, est une philosophie de vie, une attitude positive qui consiste à être bienveillant et compréhensif envers soimême dans les moments difficiles au lieu d'adopter une attitude d'autoflagellation et d'autocritique ${ }^{40}$.

Dans ce domaine, la littérature récente soutient l'idée qu'en désactivant le système de menace généré par l'objectification, l'auto-compassion constituerait un sous-facteur de la résilience émotionnelle (capacité pour un individu à faire face à une situation difficile ou génératrice de stress), favorisant une restructuration cognitive positive ${ }^{41}$. En ce sens, les recherches démontrent que le fait d'être bienveillant envers soi-même - ou auto-compassion - peut devenir un élément de résilience dans des situations de stress traumatiques ou de vulnérabilité psychologique ${ }^{42}$. De plus, ces recherches mettent en évidence les nombreuses conséquences positives de l'autocompassion. Plus spécifiquement, celle-ci est associée positivement avec une bonne santé mentale (bonheur, optimisme, émotion positive, ouverture d'esprit, initiative personnelle $\left.{ }^{43}, \ldots\right)$. La compassion pour soi-même a un impact positif sur le bien-être de la population ${ }^{44}$, entre autres des personnes âgées ${ }^{45}$ et des adolescents ${ }^{46}$. Enfin, on

${ }^{39}$ K. D. NEFF, « The development and validation of a scale to measure self-compassion », Self and Identity, 2/3, 2003, p. 223-250.

${ }^{40}$ K. D. NeFF, «Self-compassion : An alternative conceptualization of a healthy attitude toward oneself », Self and Identity, 2/2, 2003, p. 85-101.

41 P. Gilbert, K. McEwan, R. Baiño Catarino et L. Palmeira, « Fears of happiness and compassion in relationship with depression, alexithymia, and attachment security in a depressed sample », British Journal of Clinical Psychology, 53/2, 2014, p. 228-244.

42 B. L. Thompson et J. WALtz, « Self-compassion and PTSD symptom severity », Journal of Traumatic Stress, 21/6, 2008, p. 556-558.

${ }^{43}$ K. D. NefF, K. L. KirkPatrick et S. S. Rude, « Self-compassion and adaptive psychological functioning », Journal of Research in Personality, 41/1, 2007, p. 139-154 ; L. Hollis-Walker et K. Colosimo, « Mindfulness, self-compassion, and happiness in nonmeditators : A theoretical and empirical examination », Personality and Individual Differences, 50/2, 2011, p. 222-227.

${ }^{44}$ K. D. NeFF, « Self-Compassion, Self-Esteem, and Well-Being. », Social and Personality Psychology Compass, 5/1, 2011, p. 1-12.

45 A. B. Allen, E. R. Goldwasser et M. R. Leary, « Self-compassion and well-being among older adults », Self and Identity, 11/4, 2012, p. 428-453.

${ }^{46}$ K. D. NefF et P. McGehee, " Self-compassion and Psychological Resilience Among Adolescents and Young Adults », Self and Identity, 9/3, 2010, p. 225-240. 
constate son effet positif sur le stress ${ }^{47}$, la dépression ${ }^{48}$, la honte ${ }^{49}$ et l'anxiété sociale ${ }^{50}$. De fait, l'auto-compassion protège des conséquences négatives sur la santé mentale générées par l'auto-objectification. Dès lors, il paraît intéressant de se demander s'il existe des liens entre ces deux concepts.

\section{L’auto-compassion : mécanisme protecteur des conséquences négatives liées à l'auto-objectification?}

Récemment, des chercheurs se sont intéressés aux liens qui peuvent exister entre l'auto-compassion et l'image corporelle. Les recherches démontrent que les trois composantes définissant l'auto-compassion, à savoir la bienveillance envers soi-même (attitude chaleureuse et tolérante envers soi-même dans les moments douloureux, d'échec ou de sentiment d'être inadéquat(e), plutôt que d'ignorer les difficultés ou de se juger négativement ${ }^{51}$ ), la reconnaissance de son humanité (reconnaître que la souffrance et l'échec personnel font partie de l'expérience partagée par l'humanité ${ }^{52}$ ) et la pleine conscience (porter intentionnellement attention aux expériences internes ou externes du moment présent, sans formuler de jugement de valeur ${ }^{53}$ ), influencent positivement la relation entre l'image du corps et l'estime de soi ${ }^{54}$. Par exemple, la bienveillance envers soi-même, qui s'oppose à l'autocritique, joue un rôle positif dans la manière dont les femmes perçoivent leur propre corps, en adoptant un point de vue moins critique sur leur apparence. A ce sujet, l'autocritique est associée avec une fragilité de la santé mentale et un risque croissant de dépression ${ }^{55}$. Deuxièmement, la reconnaissance de son humanité induit l'idée que l'erreur est humaine et que personne n'est parfait. De fait, cette reconnaissance peut permettre aux femmes de rationaliser leurs imperfections et de ne pas se sentir isolées face à leurs expériences négatives. Enfin, la pleine conscience va permettre aux femmes de mettre en évidence leurs

47 B. L. ThOMPSON et J. WALTZ, « Self-compassion and PTSD symptom severity », op. cit.

48 P. Gilbert, M. W. Baldwin, C. Irons, J. R. Baccus et M. Palmer, « Self-Criticism and Self-Warmth : An Imagery Study Exploring Their Relation to Depression », Journal of Cognitive Psychotherapy, 20/2, 2006, p. 183-200.

49 P. Gilbert et S. Procter, « Compassionate mind training for people with high shame and self-criticism : overview and pilot study of a group therapy approach », Clinical Psychology \& Psychotherapy, 13/6, 2006, p. 353-379.

${ }^{50}$ K. H. Werner, H. Jazaieri, P. R. Goldin, M. Ziv, R. G. Heimberg, et J. J. Gross, «Self-compassion and social anxiety disorder », Anxiety, Stress \& Coping, 25/5, 2012, p. 543558.

${ }^{51}$ K. D. NEFF, « Self-compassion : An alternative conceptualization », op. cit.

52 Ibid.

53 J. KABAT-ZINN, «An outpatient program in behavioral medicine for chronic pain patients based on the practice of mindfulness meditation : Theoretical considerations and preliminary results », General Hospital Psychiatry, 4/1, 1982, p. 33-47.

${ }^{54}$ K. D. NEFF, « The development and validation of a scale to measure self-compassion », op. cit.

55 J. M. Murphy, A. A. Nierenberg, R. R. Monson, N. M. Laird, A. M. Sobol et A. H. LEIGHTON, « Self-disparagement as feature and forerunner of depression : Findings from the Stirling County Study », Comprehensive Psychiatry, 43/1, 2002, p. 13-21. 
émotions négatives afin de pouvoir les nommer, mais surtout d'aider chacune à les accepter.

En effet, si on les compare aux individus ayant un faible niveau d'auto-compassion, ceux qui sont caractérisés par un niveau d'auto-compassion élevé s'engagent moins dans des processus d'auto-surveillance, ressentent moins de honte corporelle et de dépression et sont moins concernés par les troubles alimentaires ${ }^{56}$. De plus, certains auteurs ont démontré que l'auto-compassion modère le lien entre l'image du corps et l'estime de soi chez les adolescentes ${ }^{57}$. Spécifiquement, la relation entre l'image du corps et l'estime de soi devient moins marquée chez les adolescentes qui ont un niveau élevé d'auto-compassion. De plus, l'auto-compassion génère des effets bénéfiques sur l'image corporelle, également dans les milieux cliniques. Par exemple, chez les patients souffrant de troubles alimentaires qui ont un niveau élevé d'auto-compassion en début de traitement, les conséquences négatives liées à leur alimentation s'atténuent plus vite ${ }^{58}$. En outre, l'auto-compassion semble médiatiser la relation entre l'image du corps et la détresse des femmes qui ont survécu à un cancer ${ }^{59}$. Spécifiquement, les chercheurs ont montré qu'un faible niveau d'auto-compassion est associé à un niveau élevé d'anxiété ainsi qu'à une image de soi négative chez les femmes ayant suivi un traitement complet contre le cancer (chirurgie, chimiothérapie, radiations), suggérant que l'auto-compassion pratiquée et enseignée permettrait d'améliorer la qualité de vie de ces patientes.

Récemment, des chercheurs ${ }^{60}$ ont souhaité tester directement les effets de l'autocompassion sur l'image du corps chez les femmes grâce à des séances de méditation orientée sur les trois sous-dimensions de la compassion pour soi-même. Cette pratique ancestrale offre aux pratiquants de puissants outils permettant de modifier leur perception du monde et par conséquent de renforcer leur niveau global d'autocompassion. Pour ce faire, l'équipe de chercheurs a recruté via internet (LinkedIn, Twitter, Facebook et d'autres sites liés à la perte de poids) 228 femmes âgées de 18 à 60 ans qui affirmaient être concernées par leur image corporelle. Ensuite, les chercheurs ont divisé leurs participantes en deux groupes égaux. Le premier comportait des femmes qui allaient entamer un travail de pleine conscience sous la forme de méditations guidées pendant trois semaines (groupe test). Le second comportait des

${ }^{56}$ M. Liss, et M. J. ERChull, « Not hating what you see : Self-compassion may protect against negative mental health variables connected to self-objectification in college women », Body Image, 14, 2015, p. 5-12.

${ }^{57}$ K. Pisitsungkagarn, N. Taephant, et P. Attasaranya, « Body image satisfaction and self-esteem in Thai female adolescents : the moderating role of self-compassion », International Journal of Adolescent Medicine and Health, 26/3, 2014.

58 A. C. Kelly, J. C. Carter et S. Borairi, « Are improvements in shame and selfcompassion early in eating disorders treatment associated with better patient outcomes ? ", International Journal of Eating Disorders, 47/1, 2014, p. 54-64.

59 A. Przezdziecki, K. A. Sherman, A. Baillie, A. Taylor, E. Foley, et K. StalgisBILINSKI, "My changed body : breast cancer, body image, distress and self-compassion ", Psycho-Oncology, 22/8, 201, 2013, p. 1872-1879.

${ }^{60}$ E. R. Albertson, K. D. Neff et K. E. Dill-Shackleford, « Self-Compassion and Body Dissatisfaction in Women : A Randomized Controlled Trial of a Brief Meditation Intervention », Mindfulness, 6/3, 2014, p. 444-454. 
participantes qui n'avaient rien de particulier à faire pendant ces trois semaines (groupe contrôle). Spécifiquement, les participantes dans la condition d'auto-compassion devaient écouter des enregistrements audio de méditation guidée d'une vingtaine de minutes chaque jour pendant 21 jours. Les thématiques enseignées étaient inspirées $\mathrm{du}$ « Mindful Self-Compassion Program » de Neff et Germer ${ }^{61}$. La première semaine, les exercices consistaient à amener l'auditrice à prendre conscience des états internes et externes de son propre corps de manière bienveillante et sans jugement. Lors de la deuxième semaine, au travers de séances de pleine conscience, les participantes étaient amenées à " scanner » leur corps, leurs sensations et leurs émotions tout en contrôlant leur respiration, en s'adonnant à une série d'exercices (prendre une grande respiration pour libérer toutes les tensions, apprécier chaque bouffée d'air que l'on offre à son propre corps, ...). Durant la troisième et dernière semaine, les participantes étaient amenées à adopter une perspective bienveillante sur leurs expériences de souffrances. Avant et après les trois semaines, les participantes des deux groupes ont complété des échelles mesurant l'auto-compassion, l'appréciation du corps, la honte corporelle et la dépréciation du corps. Au terme de l'expérience, les chercheurs ont mis en évidence que l'écoute de podcasts de méditation de pleine conscience guidée a un impact positif sur l'auto-compassion ainsi que sur la manière dont les femmes perçoivent leur propre corps. Spécifiquement, la pratique de la méditation joue un rôle bénéfique sur les dimensions sous-jacentes de l'auto-compassion, à savoir la bienveillance envers soi-même, l'auto-jugement, la reconnaissance de son humanité, l'isolation, la pleine conscience, sur l'identification ainsi que sur les dimensions liées à l'image du corps, comme la réduction du sentiment de honte corporelle ou l'insatisfaction par rapport à leur propre corps. Récemment, d'autres chercheurs ont confirmé des effets bénéfiques similaires de l'auto-compassion sur l'image du corps via la pratique de la pleine conscience grâce à des séances de yoga ${ }^{62}$.

\section{L'auto-compassion : un souffle de vie pour les personnes souffrant d'une image corporelle défavorable?}

En guise de conclusion, on peut dire que les recherches récentes examinant les liens entre l'auto-compassion et l'image corporelle sont prometteuses et encourageantes. Plus particulièrement, la compassion pour soi-même semble démontrer son importance dans le mécanisme de la résilience face à l'objectification sexuelle dans notre société contaminée par l'omniprésence des représentations féminines et masculines objectifiantes, trompeuses et non représentatives de sa population. Enfin, la pratique régulière d'activités renforçant le niveau d'auto-compassion (méditation, pleine conscience, yoga, développement personnel, ...) semble être une piste utile et bienveillante pour favoriser une meilleure acceptation de son corps et moins ressentir ainsi les pressions exercées par la société actuelle.

${ }^{61}$ K. D. NefF et C. K. Germer, «A pilot study and randomized controlled trial of the mindful self-compassion program », Journal of Clinical Psychology, 69/1, 2013, p. 28-44.

${ }^{62}$ A. E. Cox, S. Ullrich-French, A. N. Cole et M. D'Hondt-Taylor, « The role of state mindfulness during yoga in predicting self-objectification and reasons for exercise ", Psychology of Sport and Exercise, 22, 2016, p. 321-327. 
La bienveillance est une petite lumière intérieure qui irradie et demeure toujours allumée dans l'obscurité.

C. RAMBERT

«If you talked to your friends the way you talk to your body, youd'd have no friends left at all »

Maria Hutchinson. 